\title{
Scanning holographic microscopy with resolution exceeding the Rayleigh limit of the objective by superposition of off-axis holograms
}

\author{
Guy Indebetouw, Yoshitaka Tada, Joseph Rosen, and Gary Brooker
}

\begin{abstract}
We present what we believe to be a new application of scanning holographic microscopy to superresolution. Spatial resolution exceeding the Rayleigh limit of the objective is obtained by digital coherent addition of the reconstructions of several off-axis Fresnel holograms. Superresolution by holographic superposition and synthetic aperture has a long history, which is briefly reviewed. The method is demonstrated experimentally by combining three off-axis holograms of fluorescent beads showing a transverse resolution gain of nearly a factor of 2. () 2007 Optical Society of America

OCIS codes: $\quad 090.0090,180.0180,180.2520,110.0180,110.6880$.
\end{abstract}

\section{Introduction}

Extending the resolution beyond the theoretical Rayleigh limit of the objective has been, and still is, an important quest in high-resolution microscopy. In the recent past, many different approaches toward achieving this goal have been proposed and demonstrated. A number of superresolution methods are based on the rearrangement and smart use of the degrees of freedom of the image and of the optical system. ${ }^{1-6}$ The general idea is to utilize degrees of freedom that are deemed unnecessary. ${ }^{7-11}$ The degrees of freedom that can be sacrificed to improve the spatial resolution are numerous. For example, they can be dimensional, ${ }^{12}$ temporal, ${ }^{13,14}$ or polarization. ${ }^{15}$ Different approaches to superresolution are based on analytic extrapolation, iterative use of a priori knowledge, ${ }^{16,17}$ or deconvolution. ${ }^{18}$ Closest to the method described in this paper, structured illumination, spa-

G. Indebetouw (gindebet@vt.edu) and Y. Tada are with the Department of Physics, Virginia Tech, Blacksburg, Virginia 240610435, USA. J. Rosen and G. Brooker are with the Department of Biology, Integrated Imaging Center, Johns Hopkins University, Montgomery County Campus, 9605 Medical Center Drive, Rockville, Maryland 20850, USA. J. Rosen is also with the Department of Electrical and Computer Engineering, Ben-Gurion University of the Negev, P.O. Box 653, Beer-Sheva 84105, Israel, from which he is presently on leave.

Received 30 August 2006; revised 13 October 2006; accepted 16 October 2006; posted 16 October 2006 (Doc. ID 74610); published 2

February 2007.

0003-6935/07/060993-08 $\$ 15.00 / 0$

(C) 2007 Optical Society of America tial encoding, and methods based on aperture synthesis have also been successfully demonstrated for superresolution. These are based on the early idea that an object's spatial frequencies exceeding the limit of the pupil of the imaging system can be accessed by illuminating the object with gratings or interference patterns that shift the otherwise inaccessible spatial frequencies back into the pupil's transmission disk. ${ }^{19,20}$ This idea has been implemented in a large number of ways. ${ }^{21-27}$

Many superresolution methods deal with the improvement of a single planar image. For 3D specimens (as is often the case in biological studies, for example), it is thus necessary to process each axial section separately, and sequentially, which can be time consuming. Holographic methods alleviate this limitation by offering the possibility of improving the resolution of the entire $3 \mathrm{D}$ information recorded on the hologram. The idea of improving the resolution by adding holograms dates from the early developments of electronic holography. ${ }^{28,29}$ Recent advances in detector and computer power have triggered renewed interest in applying these ideas with modern digital holography. ${ }^{30-37}$

Digital holography has been shown capable of highresolution imaging of $3 \mathrm{D}$ biological specimens as well as of quantitative measurement of their phase information. ${ }^{38,39}$ However, one limitation of digital holography is that it requires a minimum degree of spatial and spectral coherence in order to encode the hologram phase in the form of interference fringes between the light scattered by the specimen and a reference wave. This necessity precludes the use of 
digital holography with fluorescent specimens. Yet modem biological studies rely heavily on specific fluorescence excitation and the emission of specific fluorescent probes. In scanning holography a phase encoding excitation pattern is scanned over the specimen in a $2 \mathrm{D}$ raster, and the hologram phase is encoded in the temporal domain. ${ }^{40}$ The method has been shown capable of reconstructing high-resolution holographic images of 3D fluorescent specimens without requiring the spatial coherence of the specimen's emission, ${ }^{41}$ as well as of measuring the phase of a specimen quantitatively. ${ }^{42}$ The method has also been shown to offer the possibility of synthesizing different point spread functions (PSF) ${ }^{43}$ and of locating small fluorescent features in 3D with submicrometer accuracy. 44

In this paper we demonstrate experimentally that synthesizing a pupil exceeding the objective's pupil disk is easily implemented in scanning holographic microscopy and leads to images of 3D fluorescent specimens reconstructed with a spatial resolution exceeding the Rayleigh limit of the objective. The results shown here are illustrative in demonstrating the principle, but do not attempt to test the limits of capability of the method. In Section 2, the basic ideas of scanning holographic microscopy are briefly reviewed and how superresolution is achieved by implementing the idea of pupil synthesis is described. Section 3 specifies the experimental setup, and an experimental demonstration is presented and discussed in Section 4. Final remarks and a summary are given in Section 5 .

\section{Theoretical Background}

In conventional scanning holography, two pupils are combined by a beam splitter and superposed in the entrance pupil of the objective. One pupil is a spherical wave filling the objective's pupil disk, and having a curvature chosen to produce, in the back focal plane of the objective (which is also the object's space), a spherical wave with a radius of curvature $z_{0}$. The other is a point at the center of the entrance pupil of the objective, producing a plane wave in the object's space. The interference of these two waves results in a 3D Fresnel zone pattern (FZP) that is scanned over the specimen in a $2 \mathrm{D}$ raster. The scattered or fluorescent light is collected by a nonimaging detector (photodiode, or photomultiplier). A single-sideband hologram can be obtained from this data in two ways: either by heterodyne detection using a frequency difference between the two pupils, ${ }^{45}$ or by homodyne detection ${ }^{46}$ using three scans with three fixed phase differences between the two pupils. ${ }^{47,48}$ Other methods of data acquisition have also been proposed. ${ }^{49}$ The hologram can be reconstructed numerically by Fresnel back propagation or by correlation with an experimental PSF. The spatial resolution of the reconstructed image is determined by the numerical aperture of the FZP scanned over the object. With an on-line hologram, the spatial frequency spectrum of the reconstruction is confined to the disk of the objective's pupil, which has a cutoff frequency
$\rho_{M A X}=N A_{O B J} / \lambda$, where $N A_{O B J}$ is the numerical aperture of the objective, and $\lambda$ is the wavelength of the radiation.

To extend the spectrum beyond the objective's cutoff in a particular direction specified by a unit vector $\hat{n}$, one can record an off-axis hologram by scanning an off-axis FZP on the specimen. If the spatial frequency offset of the FZP is $\rho_{0} \hat{n}$, the spatial frequency spectrum of the reconstruction is extended up to a value $\rho_{M A X}+\rho_{0}$ in the direction of $\hat{n}$. By combining several holograms with shifts in different angular directions, it is thus possible to tile an object's spatial frequency spectrum that extends, in principle, far beyond the objective's pupil disk.

The two pupils needed to create an off-axis FZP are

$$
\begin{aligned}
\tilde{P}_{1}(\vec{\rho}) & =\exp \left(i \pi \lambda z_{0} \rho^{2}\right) \operatorname{Disk}\left(\rho / \rho_{M A X}\right), \\
\tilde{P}_{2 j}(\vec{\rho}) & =\delta\left(\vec{\rho}-\rho_{0} \hat{n}_{j}\right) .
\end{aligned}
$$

$\operatorname{Disk}(x)=1$ for $x<1$, and $=0$ for $x>1$. In these expressions, $\vec{\rho}$ is the transverse spatial frequency vector in the pupil plane, proportional to the transverse spatial coordinate vector $\vec{r}_{P}$ in the pupil: $\vec{\rho}=\vec{r}_{P} /$ $\lambda f_{O B J}$, where $f_{O B J}$ is the focal length of the objective. ${ }^{50}$ In the object's space, $P_{1}(\vec{r})$ is a converging spherical wave with radius of curvature $z_{0}$, and $P_{2 j}(\vec{r})$ is a plane wave with a transverse spatial frequency $\rho_{0} \hat{n}_{j}$. After heterodyne detection, or phase-shifted homodyne detection, each object point is encoded as an off-axis spherical wave $S_{j}(\vec{r}, z)$, where $z$ is the axial coordinate in object space measured from the focal plane of the objective. In Fourier space, we have

$$
\begin{aligned}
\tilde{S}_{j}(\vec{\rho}, z)= & \tilde{P}_{1}(\vec{\rho}, z) \oplus \tilde{P}_{2 j}(\vec{\rho}, z) \\
= & \exp \left\{i \pi \lambda\left[z_{0} \rho_{0}^{2}+\left(z_{0}-z\right)\left(\rho^{2}-2 \vec{\rho} \cdot \rho_{0} \hat{n}_{j}\right)\right]\right\} \\
& \times \operatorname{Disk}\left(\left|\vec{\rho}-\rho_{0} \hat{n}_{j}\right| / \rho_{\text {MAX }}\right),
\end{aligned}
$$

where $\oplus$ symbolizes a correlation integral, and $\tilde{P}_{1,2}(\vec{\rho}, z)$ are the generalized (defocused) pupils ${ }^{51}$ defined as

$$
\tilde{P}_{1,2}(\vec{\rho}, z)=\tilde{P}_{1,2}(\vec{\rho}) \exp \left(i 2 \pi z \sqrt{\lambda^{-2}-\rho^{2}}\right),
$$

or in paraxial approximation,

$$
\begin{aligned}
\tilde{P}_{1}(\vec{\rho}, z)= & \exp (i 2 \pi z / \lambda) \exp \left[i \pi \lambda\left(z_{0}-z\right) \rho^{2}\right] \\
& \times \operatorname{Disk}\left(\rho / \rho_{\text {MAX }}\right), \\
\tilde{P}_{2 j}(\vec{\rho}, z)= & \exp (i 2 \pi z / \lambda) \exp \left(-i \pi \lambda z \rho_{0}^{2}\right) \delta\left(\vec{\rho}-\rho_{0} \hat{n}_{j}\right) .
\end{aligned}
$$

The Fourier transform of the specimen hologram is given by

$$
\tilde{H}_{O j}=\int \mathrm{d} z \tilde{I}(\vec{\rho}, z) \tilde{S}_{j}(\vec{\rho}, z)
$$


where $\tilde{I}(\vec{\rho}, z)$ is the 2D transverse Fourier transform of the 3D object intensity distribution $I(\vec{r}, z)$. The reconstruction of the hologram in a chosen axial plane of focus $z=z_{R}$ can be obtained by digital Fresnel back propagation from the hologram for a distance $z_{0}+z_{R}$. In the experiment discussed in Section 4, we chose to reconstruct the hologram by correlation with the experimental hologram of a subresolution point source. As has been shown previously, ${ }^{41}$ this method offers a way of reducing the phase aberrations of the objective. ${ }^{41}$ With high NA objectives, these aberrations are attributable to the fact that the objective is not (and cannot be) used in the geometry for which it was designed, and can be severe. In the experiment described in Section 3, this scheme is implemented by recording a reference hologram $H_{R j}(\vec{r})$ of a point source $\delta(\vec{r}, z)$, and propagating it to the desired reconstruction plane by using a propagation factor $P_{j}\left(\vec{r}, z_{R}\right)$. In Fourier space, we have

$$
\begin{aligned}
\tilde{H}_{R j}(\vec{\rho})= & \exp \left[i \pi \lambda z_{0}\left(\left|\vec{\rho}-\rho_{0} \hat{n}_{j}\right|^{2}\right)\right] \\
& \times \operatorname{Disk}\left(\left|\vec{\rho}-\rho_{0} \hat{n}_{j}\right| / \rho_{M A X}\right), \\
\tilde{P}_{j}\left(\vec{\rho}, z_{R}\right)= & \exp \left[-i \pi \lambda z_{R}\left(\rho^{2}-2 \vec{\rho} \cdot \rho_{0} \hat{n}_{j}\right)\right] .
\end{aligned}
$$

The Fourier transform of the reconstructed image is then given by

$$
\begin{aligned}
\tilde{R}_{j}\left(\vec{\rho}, z_{R}\right)= & \tilde{H}_{O j}(\vec{\rho})\left[\tilde{H}_{R j}(\vec{\rho}) \tilde{P}_{j}\left(\vec{\rho}, z_{R}\right)\right]^{*} \\
= & \int \mathrm{d} z \tilde{I}(\vec{\rho}, z) \exp \left[i \pi \lambda\left(z_{R}-z\right)\left(\rho^{2}-2 \vec{\rho} \cdot \rho_{0} \hat{n}_{j}\right)\right] \\
& \times \operatorname{Disk}\left(\left|\vec{\rho}-\rho_{0} \hat{n}_{j}\right| / \rho_{M A X}\right),
\end{aligned}
$$

where the asterisk represents a complex conjugation. In the plane of focus $z=z_{R}$, the reconstruction has a spatial frequency spectrum consisting of the object's spatial frequencies located within a disk of radius $\rho_{\text {MAX }}$, centered at $\rho_{0} \hat{n}_{j}$. By adding coherently the reconstruction amplitudes of a number of holograms with different spatial frequency shifts, one can, in principle, tile a synthetic pupil with an area far exceeding the pupil disk of the objective.

It must be emphasized that although the pupil representation in Eq. (1) is, strictly speaking, valid only in the paraxial regime, ${ }^{52}$ the method of scanning holographic microscopy is not limited to small NAs. In fact, since we actually measure the PSF, and reconstruct the holograms by this experimental PSF, our method is valid regardless of the system's NA, and it is independent of the theoretical representation of the PSF.

\section{Experimental Arrangement}

The experimental setup of a scanning holographic microscope has been described in detail in previous publications. ${ }^{40,41}$ For completeness, a sketch is given in Fig. 1. The only addition to the previous arrangements is the introduction of a wedge prism in a conjugate object plane to shift the spatial frequency of the plane wave creating the FZP by a chosen amount in a chosen direction. The wedge is then rotated in discrete angular steps to cover a desired area of the object's spatial frequency spectrum. For experimental simplicity we chose to introduce both the spherical wave and the off-axis plane wave forming the FZP through the pupil of the objective. With this method, the largest spatial frequency shift achievable is the objective's frequency cutoff, namely, $\rho_{0} \leq \rho_{M A X}$. Opting for this geometry is not a necessary requirement.

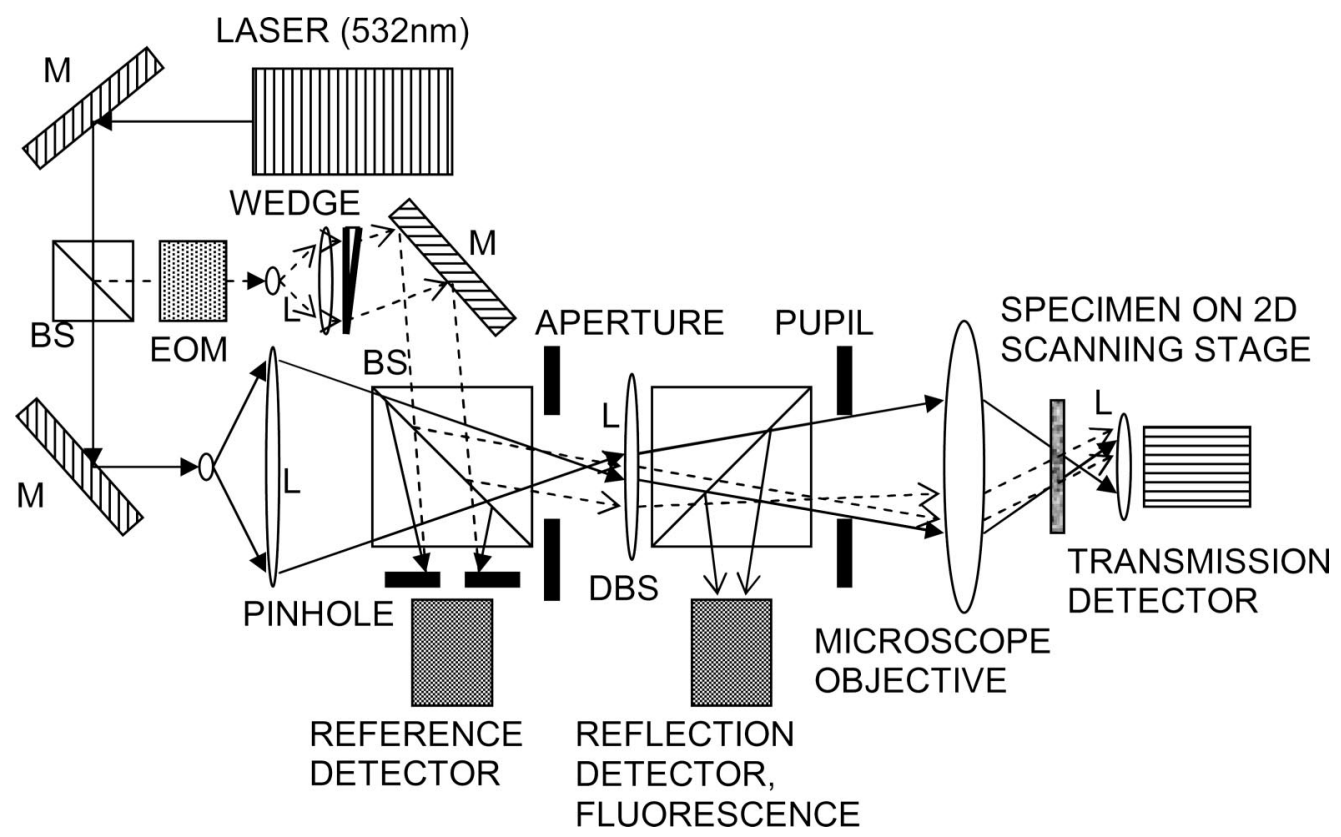

Fig. 1. Schematic of an off-axis scanning holographic microscope. M, mirrors; BS, beam splitters; DBS, dichroic beam splitter; EOM, electro-optic phase modulator. $\mathrm{L}_{1,2}$ are achromat doublet lenses, $16 \mathrm{~cm}$ focal length, $\mathrm{L}_{3}$ is a collecting lens, $1 \mathrm{~cm}$ focal length. The wedge on a rotating stage is used to create off-axis Fresnel patterns on the specimen. 


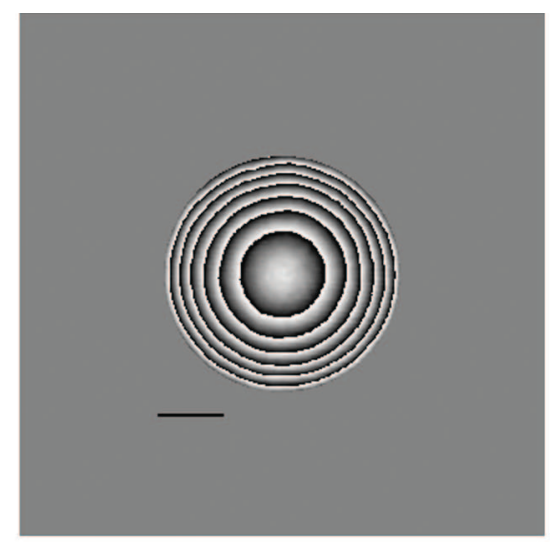

(a)

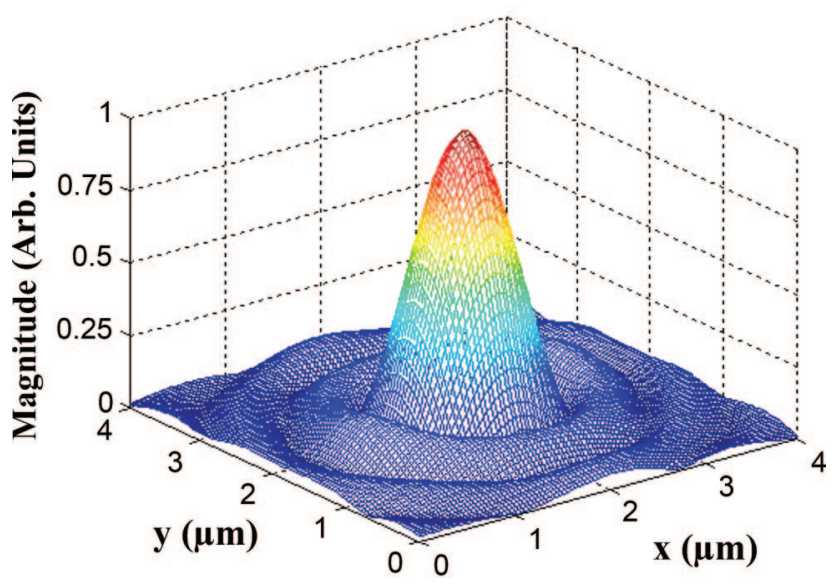

(b)

Fig. 2. (Color online) (a) Wrapped phase of the on-line hologram of a $0.5 \mu \mathrm{m}$ diameter pinhole. The scale bar is $10 \mu \mathrm{m}$. The phase distribution has a radius $\sim 18 \mu \mathrm{m}$, a Fresnel number $\sim 12$, and a radius of curvature $\sim 50 \mu \mathrm{m}$. (b) Amplitude of the reconstruction of the $0.5 \mu \mathrm{m}$ pinhole using the on-line hologram. FWHM $\sim 1.0 \mu \mathrm{m}$.

In principle, it is possible to introduce the off-axis plane waves externally at any angle, although this practice may be difficult to implement at high NA. Nevertheless, the transfer function of such system could in principle be extended to its theoretical frequency limit of $2 / \lambda$ and cover the entire spectral region inside this limit. Additionally, it may also be possible to introduce several plane waves simultaneously by using an array of sources. ${ }^{35-37}$

The experiment uses a Mitutoyo Plan Apo objective 20x, 0.42 NA, chosen for its long working distance. The detector is a commercial Hamamatsu photomultiplier tube (R1166). The specimen is scanned in a 2D raster using a piezo scanning stage (PI Hera 625). The FZP modulation is provided by an electro-optic phase modulator (Linos LM0202) driven by a saw tooth waveform at $25 \mathrm{kHz}$. The signal is sampled at $100 \mathrm{kSample} / \mathrm{s}$ by a GageScope 1602. The hologram is constructed line by line after bandpass filtering at the modulation frequency, as previously described, and reconstructed digitally using MATLAB codes (MATLAB 7.1.0.144/R14 SP3) on a standard personal computer (PC).
It was found that at high NA, the fact that the off-axis FZP has to cross the cover glass of the specimen before illuminating it introduced a fair amount of aberrations and distortions. Furthermore it also changed the spatial frequency offset by an amount difficult to predict. In principle, if the optical properties of the specimen and its mounting environment are known, this effect can be compensated for numerically. We chose to eliminate these difficulties by using a specimen without cover glass. The specimen used in the demonstration in Section 4 is a cluster of $1 \mu \mathrm{m}$ diameter fluorescent beads (R0100 Polymer Microspheres, Duke Scientific, Palo Alto, Calif., USA) smeared on a microscope slide without a cover slip.

\section{Experimental Results}

To assess the gain in the resolution of the system, we used the reconstruction of a subresolution pinhole $(0.5 \mu \mathrm{m}$ diameter) to estimate the size of the PSF. With a NA of 0.42 , the theoretical Rayleigh resolution limit of the objective is $1.22 \lambda_{E M} / 2 N A \approx 0.9 \mu \mathrm{m}$. $\lambda_{E M}$ $=600 \mathrm{~nm}$ is the emission wavelength of the beads. The wrapped phase of the on-line hologram of the

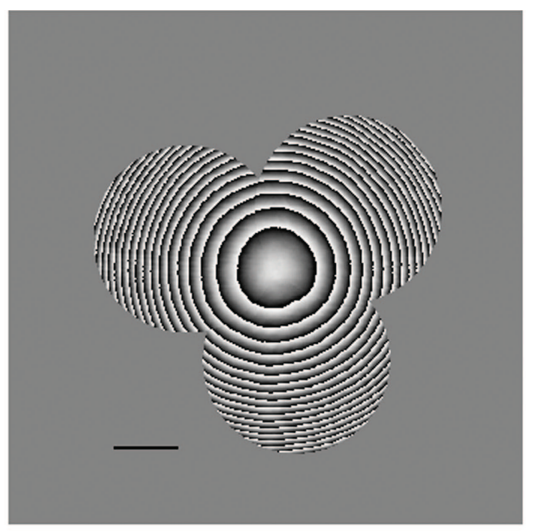

(a)

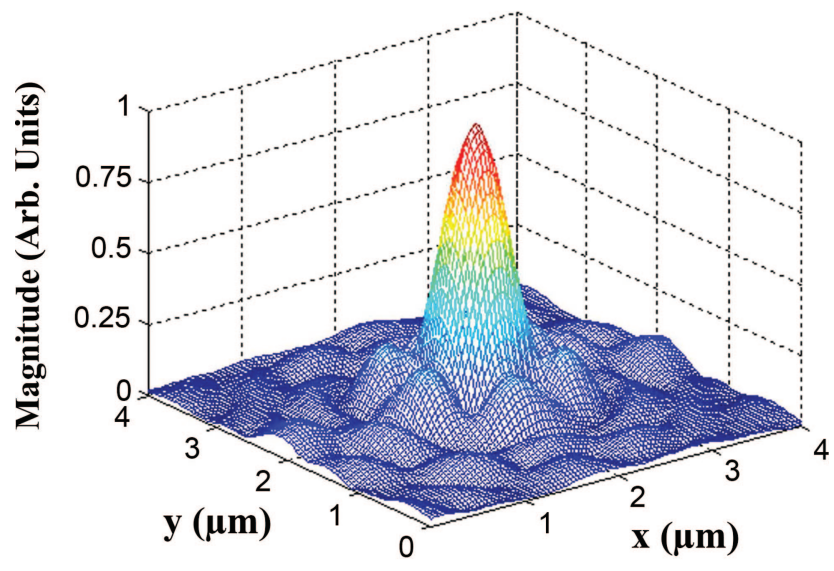

(b)

Fig. 3. (Color online) (a) Wrapped phase of three off-axis holograms of the $0.5 \mu \mathrm{m}$ pinhole illustrating the idea of pupil synthesis. The scale bar is $10 \mu \mathrm{m}$. (b) Amplitude of the reconstruction of the $0.5 \mu \mathrm{m}$ pinhole using the composite off-axis holograms. FWHM $\sim 0.7 \mu \mathrm{m}$. 


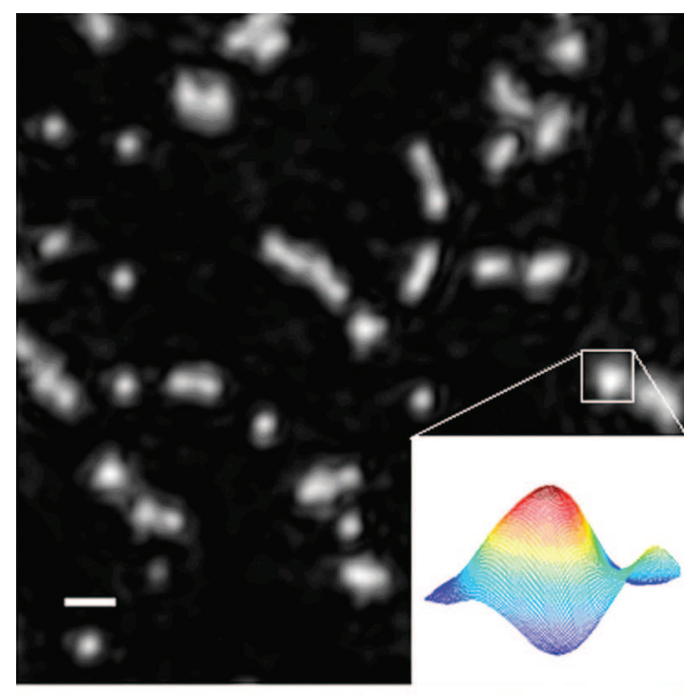

(a)

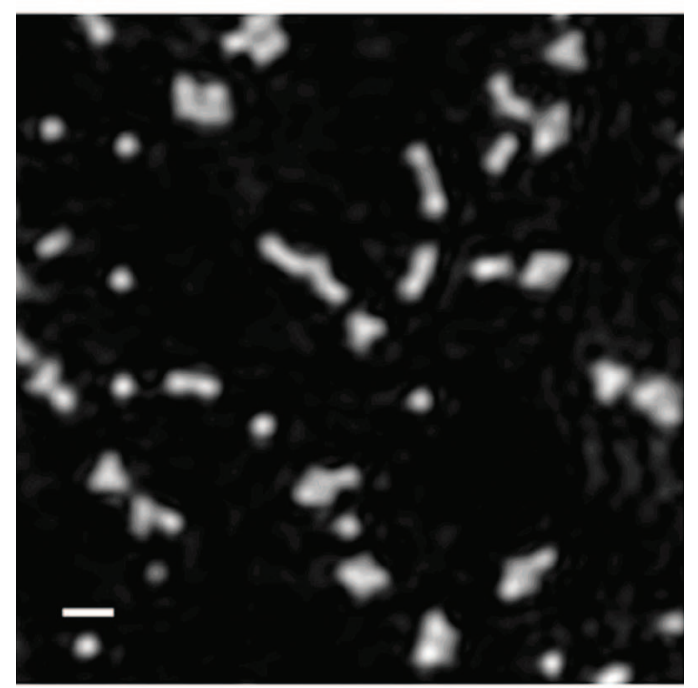

(b)

Fig. 4. (Color online) (a) Reconstruction of the on-axis hologram of a collection of $\sim 1.0 \mu \mathrm{m}$ fluorescent beads (excitation/emission wavelengths $=532 \mathrm{~nm} / 600 \mathrm{~nm}$ ) at the best focus distance of $47.5 \mu \mathrm{m}$ from the focal plane of the objective. The scale bar is $5 \mu \mathrm{m}$. Bead clusters are just barely resolved. (b) Same reconstruction at a focus distance of $49 \mu \mathrm{m}$. The two planes are within the Rayleigh range of the on-axis scanning FZP.

$0.5 \mu \mathrm{m}$ diameter pinhole is shown in Fig. 2(a), and its reconstruction is shown in Fig. 2(b). The hologram has a Fresnel number $F \approx 12$, and a radius $a \approx 18 \mu \mathrm{m}$. Using the relation $F=a^{2} / \lambda_{E X} z_{0}$ with an excitation wavelength $\lambda_{E X}=532 \mathrm{~nm}$, the radius of curvature of the hologram is found to be $z_{0} \approx$ $50 \mu \mathrm{m}$, and its equivalent NA is $a / z_{0} \approx 0.35$. This leads to an expected theoretical Rayleigh limit $\approx 0.9 \mu \mathrm{m}$, which is close to the Rayleigh resolution of the objective. The observation that the smaller equivalent NA of the hologram leads to the same resolution limit as that of the objective is attributable to the fact that the objective forms an image at the emission wavelength while the hologram is formed at the

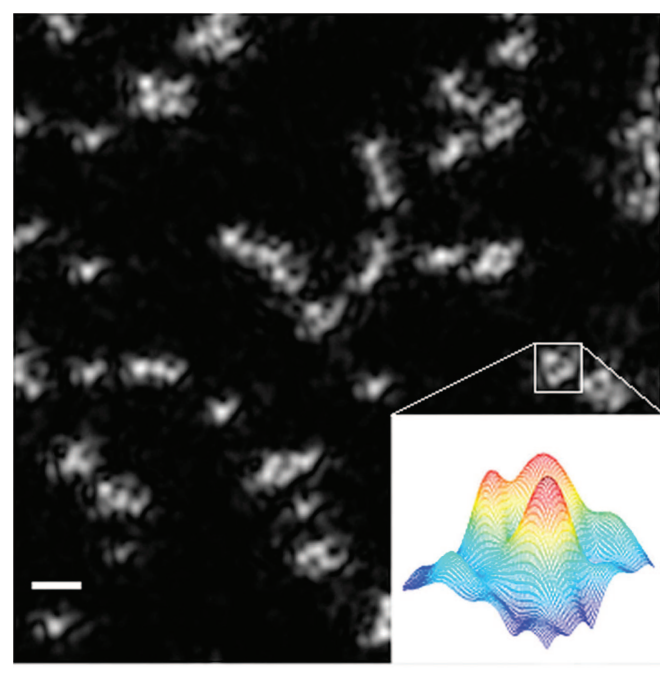

(a)

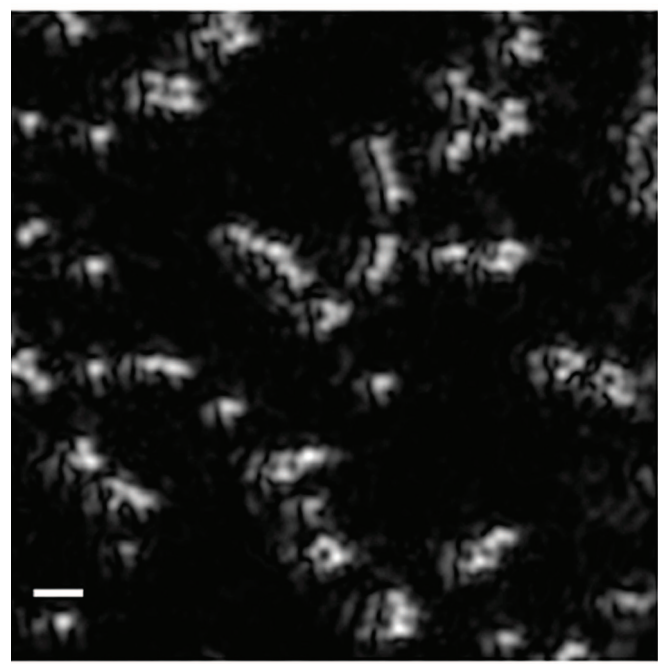

(b)

Fig. 5. (Color online) Coherent sum of the complex amplitudes of the reconstructions of three off-axis holograms recorded with off sets $120^{\circ}$ apart. The scale bar is $5 \mu \mathrm{m}$. (a) Best focus at $47.5 \mu \mathrm{m}$ from the focal plane of the objective. (b) Same reconstruction at a focus distance of $49 \mu \mathrm{m}$. The distance between the two planes is close to the Rayleigh range of the synthesized FZP, and different bead clusters are focused in different planes.

shorter excitation wavelength. A dye with a larger Stokes shift would lead to an even larger gain. The reconstruction shown in Fig. 2(b) has a FWHM equal to $\approx 1.0 \mu \mathrm{m}$. Since this is the convolution of the actual PSF with the $0.5 \mu \mathrm{m}$ pinhole, the size of the experimental PSF is estimated to be $\approx 0.9 \mu \mathrm{m}$, or smaller. Three off-axis holograms of the pinhole were recorded with a spatial frequency offset $\rho_{0} \approx$ $\rho_{M A X} \approx 0.66 \mu \mathrm{m}^{-1}$ in directions $120^{\circ}$ apart. The wrapped phases of the three holograms are combined in Fig. 3(a) to illustrate the wider spatial frequency coverage of the composite hologram. The reconstruction of the pinhole from this hologram is shown in Fig. 3(b). Its FWHM is $\approx 0.7 \mu \mathrm{m}$. The actual size of the PSF of the composite hologram is thus estimated 


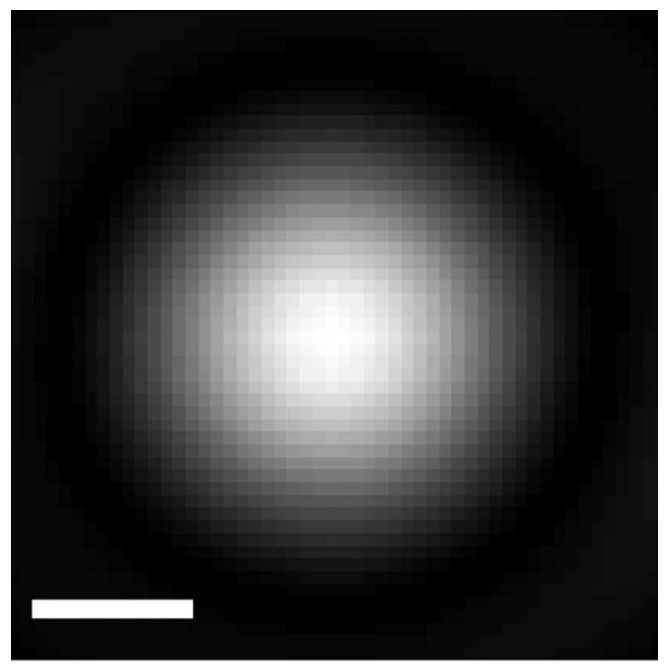

(a)

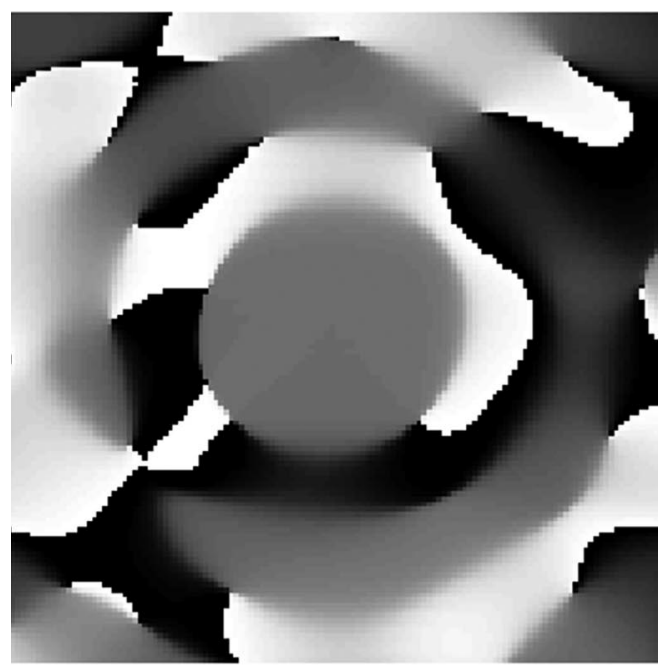

(b)

Fig. 6. (a) Absolute value and (b) wrapped phase of the reconstruction of a $0.5 \mu \mathrm{m}$ diameter pinhole from the on-axis hologram. The phase profile is typical of an Airy pattern with a central lobe diameter $\approx 1.5 \mu \mathrm{m}$. The scale bar is $1 \mu \mathrm{m}$.

to be smaller than $\approx 0.6 \mu \mathrm{m}$, which corresponds to an equivalent NA larger than $\approx 0.54$. The reduction of the resolution limit by a factor $\sim 0.6$ or smaller could be further improved by combining more than three holograms. If the off-axis FZP are introduced on the specimen through the objective, as done in the experiment, we can expect a resolution limit down to a factor $\sim 0.5$ that of the objective. Further improvement is possible in principle down to the theoretical limit $\lambda / 2$, by introducing off-axis FZP externally (albeit this may involve some technical difficulties).

To demonstrate the reality of the achieved resolution improvement, we chose a sample consisting of fluorescent beads with a diameter slightly larger than the $\approx 0.9 \mu \mathrm{m}$ resolution limit of the objective at the emission wavelength, and slightly larger than the $\approx 0.9 \mu \mathrm{m}$ resolution limit of the on-axis hologram at the excitation wavelength. The expectation is that

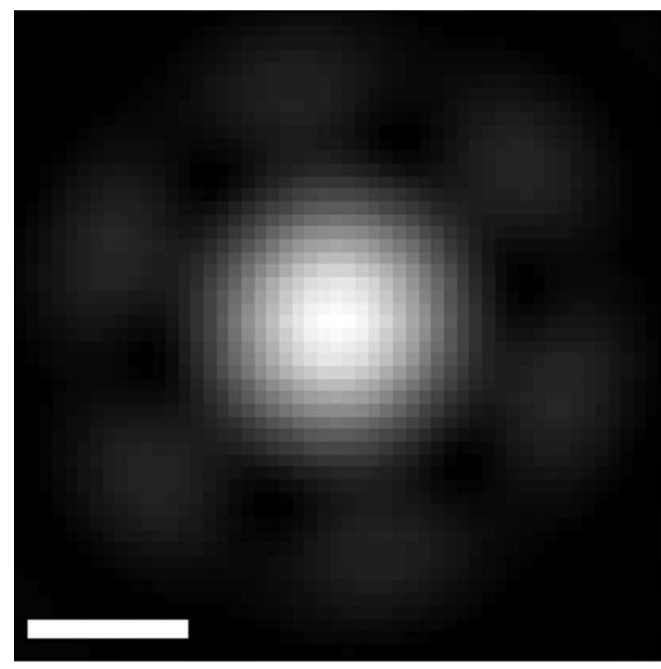

(a)

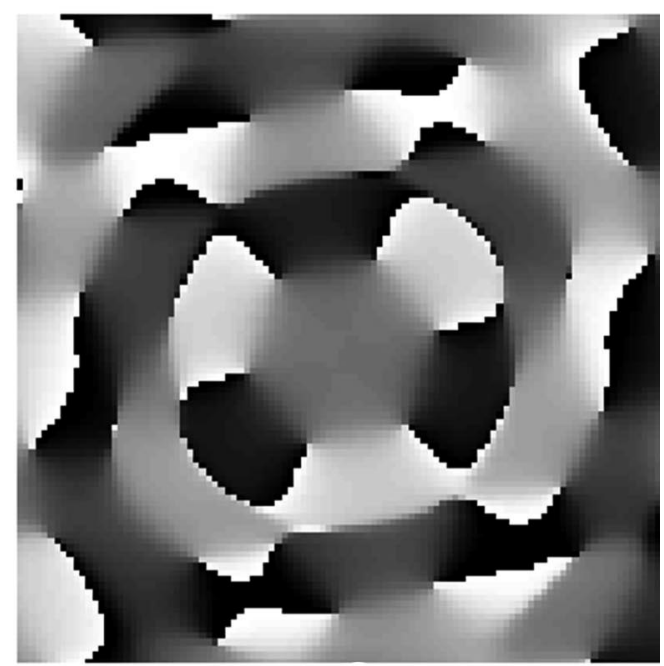

(b)

Fig. 7. (a) Absolute value and (b) wrapped phase of the coherent sum of the reconstructions of a $0.5 \mu \mathrm{m}$ diameter pinhole from the three off-axis holograms. The threefold symmetry of the destructive interference results in a narrower amplitude distribution $(\mathrm{a})$, and a central lobe diameter $\approx 1.0 \mu \mathrm{m}$. The scale bar is $1 \mu \mathrm{m}$.

the reconstruction of the on-axis hologram will show, at best, barely unresolved beads, while the composite reconstruction should reveal beads that are resolved. The three holograms of the fluorescent beads were recorded in reflection, reconstructed individually, and the amplitudes of their reconstructions were added coherently. Figures 4(a) and 4(b) show the reconstructions of the on-axis hologram in two different planes. As expected, individual beads are detected in the best plane of focus $[z=47.5 \mu \mathrm{m}$ in Fig. 4(a) $]$, but the clusters are not resolved. Shifting the focal plane to $z=49 \mu \mathrm{m}$ does not reveal anything different because the two planes with an axial separation of $1.5 \mu \mathrm{m}$ are well within the Rayleigh focal distance of $\approx 3.5 \mu \mathrm{m}$. The composite reconstructions of the three off-axis holograms in the same two focal planes are 
shown in Figs. 5(a) and 5(b). The clusters of beads are now well resolved. Furthermore Figs. 5(a) and 5(b) reveal that different clusters are in best focus in different planes. This is to be expected since the two planes are $1.5 \mu \mathrm{m}$ apart, while the axial Rayleigh distance of the composite hologram is $\approx 1.8 \mu \mathrm{m}$.

It is interesting to observe how the gain in transverse and axial resolution is a result of the mutual interference of the complex amplitudes of the reconstructions of the off-axis holograms. This is illustrated in Figs. 6 and 7, which show the absolute values and the wrapped phases of the reconstructions of the $0.5 \mu \mathrm{m}$ pinhole for the on-axis hologram (Fig. 6 ), and for the three combined off-axis holograms (Fig. 7). The middle gray in the phase figures represents a phase zero, while the black and the white represent a phase $-\pi$ and $+\pi$, respectively. Figure 6 is that of a typical Airy pattern. Combining the complex amplitudes of the three off-axis holograms leads to destructive interferences at the rim of the main lobe of the Airy disc, leading to a narrowing of its size. The threefold symmetry of the destructive interferences is clearly identified in Fig. 7.

\section{Summary}

We have shown experimentally that it is possible to overcome the spatial resolution limit of the objective in holographic microscopy by combining a number of off-axis scanning holograms to synthesize a pupil area larger than that of the objective. The principle of superresolution by holographic superposition and synthetic aperture has a long history, which is briefly reviewed. The implementation of this principle using incoherent holographic scanning microscopy is demonstrated by using holograms of fluorescent beads.

This research was funded in part by a grant from the National Institute of Health, Office of Extramural Research, grant R21 RR018440, and by a grant from the National Science Foundation, grant DBI0420382. J. Rosen's research was supported in part by the Israel Sciences Foundation, grant 119-03.

\section{References}

1. G. Toraldo di Francia, "Super-gain antennas and optical resolving power," Nuovo Cimento, Suppl. 9, 426-438 (1952).

2. G. Toraldo di Francia, "Resolving power and information," J. Opt. Soc. Am. 45, 497-501 (1955).

3. G. Toraldo di Francia, "Degrees of freedom of an image," J. Opt. Soc. Am. 59, 799-804 (1969).

4. W. Lukosz, "Optical systems with resolving power exceeding the classical limits," J. Opt. Soc. Am. 56, 1463-1472 (1966).

5. W. Lukosz, "Optical systems with resolving power exceeding the classical limits, II,” J. Opt. Soc. Am. 57, 932-941 (1967).

6. I. J. Cox and J. R. Sheppard, "Information capacity and resolution in an optical system," J. Opt. Soc. Am. A 3, 1152-1158 (1986).

7. D. Mendlovic and A. W. Lohmann, "Space-bandwidth product adaptation and its application to superresolution: fundamentals," J. Opt. Soc. Am. A 4, 558-562 (1997).

8. D. Mendlovic, A. W. Lohmann, and Z. Zalevsky, "Spacebandwidth product adaptation and its application to superresolution: examples," J. Opt. Soc. Am. A 4, 563-567 (1997).

9. Z. Zalevsky, D. Mendlovics, and A. W. Lohmann, "Optical systems with improved resolving power," in Progress in $\mathrm{Op}$ tics, E. Wolf, ed. (Elsevier, 2000), Vol. 40, pp. 271-341.

10. T. Leizerson, S. G. Lipson, and V. Sarafi, "Superresolution in far-field imaging," J. Opt. Soc. Am. A 19, 436-443 (2002).

11. M. A. Grimm and A. W. Lohmann, "Superresolution image for one-dimensional objects,” J. Opt. Soc. Am. 56, 1151-1156 (1966).

12. D. Mendlovics, A. W. Lohmann, N. Konforti, I. Kiryuschev, and Z. Zalevsky, "One-dimensional superresolution optical system for temporally restricted objects," Appl. Opt. 36, 2353-2359 (1997).

13. A. Shemer, D. Mendlovics, Z. Zalevsky, J. Garcia, and P. Garcia-Martinez, "Superresolving optical system with time multiplexing and computer decoding," Appl. Opt. 38, 72457251 (1999).

14. A. W. Lohmann and D. P. Paris, "Superresolution for nonbirefringent objects," Appl. Opt. 3, 1037-1043 (1964).

15. E. N. Leith, D. Angell, and C. P. Kuei, "Superresolution by incoherent to coherent conversion," J. Opt. Soc. Am. A 4, 10501054 (1987).

16. R. W. Gerchberg, "Super-resolution through error energy reduction," Opt. Acta 21, 709-720 (1974).

17. M. Bertero and C. De Mol, "Superresolution by data inversion," in Progress in Optics, E. Wolf, ed. (Elsevier, 1996), Vol. 36, pp. 129-178.

18. B. Colicchio, O. Haeberle, C. Xu, A. Dieterlen, and G. Jung, "Improvement of the LLS and MAP deconvolution algorithms by automatic determination of optimal regularization parameters and prefiltering of original data," Opt. Commun. 244, 37-49 (2005).

19. P. C. Sun and E. N. Leith, "Superresolution by spatialtemporal encoding methods,” Appl. Opt. 31, 4857-4862 (1992).

20. M. G. L. Gustafsson, "Surpassing the lateral resolution limit by a factor of two using structured illumination microscopy," J. Microsc. 198, 82-87 (2000).

21. M. A. A. Neil, R. Juskaitis, and T. Wilson, "Method of obtaining optical sectioning by using structured light in a conventional microscope," Opt. Lett. 22, 1905-1907 (1997).

22. J. T. Frohn, H. F. Knapp, and A. Stemmer, "Three-dimensional resolution enhancement in fluorescence microscopy by harmonic excitation," Opt. Lett. 26, 828-830 (2001).

23. J. Garcia, Z. Zalevsky, and D. Fixler, "Synthetic aperture superresolution by speckle pattern projection," Opt. Express 13, 6073-6078 (2005).

24. O. Haeberle and B. Simon, "Improving the lateral resolution in confocal fluorescence microscopy using laterally interfering excitation beams," Opt. Commun. 259, 400-408 (2006).

25. M. Martinez-Corral, P. Andres, C. J. Zapata-Rodriguez, and M. Kowalczyk, "Three-dimensional superresolution by annular binary filters," Opt. Commun 165, 267-278 (1999).

26. M. Gu, T. Tannous, and C. R. J. Sheppard, "Improved axial resolution in focal fluorescence microscopy with annular pupils," Opt. Commun. 110, 533-539 (1994).

27. M. Martinez-Corral, M. T. Caballero, E. H. K. Stelzer, and J. Swoger, "Tailoring the axial shape of the point spread function using the Toraldo concept," Opt. Express 10, 98-103 (2002).

28. J. W. Goodman and R. W. Lawrence, "Digital image information from electronically detected holograms," Appl. Phys. Lett. 11, 77-79 (1967).

29. T. Sato, M. Ueda, and G. Yamagishi, "Superresolution microscope using electrical superposition of holograms," Appl. Opt. 13, 406-408 (1973).

30. M. Ueda, T. Sato, and M. Kondo, "Superresolution by multiple superposition of images holograms having different carrier frequencies," Opt. Acta 20, 403-410 (1973).

31. X. Chen and S. R. Brueck, "Imaging interferometric lithography approaching the resolution limit of the optics," Opt. Lett. 24, 124-126 (1999).

32. F. Le Clerc, M. Gross, and L. Collot, "Synthetic-aperture ex- 
periment in the visible with on-axis digital heterodyne holography," Opt. Lett. 26, 1550-1552 (2001).

33. J. R. Hassig, "Digital off-axis holography with synthetic aperture," Opt. Lett. 27, 2179-2181 (2002).

34. C. J. Schwarz, Y. Kuznetsova, and S. R. J. Brueck, "Imaging interferometric microscopy," Opt. Lett. 28, 1424-1426 (2003).

35. V. Mico, Z. Zalevsky, P. Garcia-Martinez, and J. Garcia, "Single step superresolution by interferometric imaging," Opt. Express 12, 2589-2596 (2004).

36. V. Mico, Z. Zalevsky, and J. Garcia, "Superesolution optical system by common path interferometry," Opt. Express 14, 5168-5177 (2006).

37. V. Mico, Z. Zalevsky, P. Garcia-Martinez, and J. Garcia, "Superresolved imaging in digital holography by superposition of tilted wavefronts," Appl. Opt. 45, 822-828 (2006).

38. E. Cuche, F. Bevilacqua, and C. Depeursinge, "Digital holography for quantitative phase contrast imaging," Opt. Lett. 24, 291-293 (1999).

39. E. Cuche, P. Marquet, and C. Depeursinge, "Simultaneous amplitude-contrast and quantitative phase-contrast microscopy by numerical reconstruction of Fresnel off-axis holograms," Appl. Opt. 38, 6994-7001 (1999).

40. G. Indebetouw, A. El Maghnouji, and R. Foster, "Scanning holographic microscopy with transverse resolution exceeding the Rayleigh limit and extended depth of focus," J. Opt. Soc. Am. A 22, 892-898 (2005).

41. G. Indebetouw and W. Zhong, "Scanning holographic microscopy of three-dimensional fluorescent specimens," J. Opt. Soc. Am. A 23, 1699-1707 (2006).

42. G. Indebetouw, Y. Tada, and L. Leacock, "Quantitative phase imaging with scanning holographic microscopy: experimental assessment," Biomed. Eng. Online 5, doi:10.1186/1475-925x5-63 (2006).

43. G. Indebetouw, W. Zhong, and D. Chamberlin-Long, "Pointspread function synthesis in scanning holographic microscopy," J. Opt. Soc. Am. A 23, 1708-1717 (2006).

44. G. Indebetouw, "A posteriori quasi-sectioning of the threedimensional reconstructions of scanning holographic microscopy," J. Opt. Soc. Am. A 23, 2657-2661 (2006).

45. G. Indebetouw, P. Klysubun, T. Kim, and T.-C. Poon, "Imaging properties of scanning holographic microscopy," J. Opt. Soc. Am. A 17, 380-390 (2000).

46. J. Rosen, G. Indebetouw, and G. Brooker, "Homodyne scanning holography," Opt. Express 14, 4280-4285 (2006).

47. I. Yamaguchi and T. Zhang, "Phase-shifting digital holography," Opt. Lett. 22, 1268-1270 (1997).

48. I. Yamaguchi, J.-I. Kato, S. Ohta, and J. Mizuno, "Image formation in phase-shifting digital holography and application to microscopy," Appl. Opt. 40, 6177-6186 (2001).

49. J. Swoger, M. Martinez-Corral, J. Huysken, and E. H. K. Stelzer, "Optical scanning holography as a technique for high-resolution three-dimensional biological microscopy," J. Opt. Soc. Am. A 19, 1910-1918 (2002).

50. J. W. Goodman, Introduction to Fourier Optics, 2nd ed. (McGraw-Hill, 1966).

51. C. W. McCutchen, "Generalized aperture and threedimensional diffraction images," J. Opt. Soc. Am. 54, 240-244 (1964).

52. M. Gu, Advance in Optical Imaging Theory (Springer, 2000). 